\title{
Eigenvectors of the Discrete Fourier Transform Based on the Bilinear Transform
}

\author{
Ahmet Serbes and Lutfiye Durak-Ata (EURASIP Member) \\ Department of Electronics and Communications Engineering, Yildiz Technical University, Yildiz, Besiktas, 34349, Istanbul, Turkey \\ Correspondence should be addressed to Ahmet Serbes, ahmet.serbes@gmail.com
}

Received 19 February 2010; Accepted 24 June 2010

Academic Editor: L. F. Chaparro

Copyright () 2010 A. Serbes and L. Durak-Ata. This is an open access article distributed under the Creative Commons Attribution License, which permits unrestricted use, distribution, and reproduction in any medium, provided the original work is properly cited.

\begin{abstract}
Determining orthonormal eigenvectors of the DFT matrix, which is closer to the samples of Hermite-Gaussian functions, is crucial in the definition of the discrete fractional Fourier transform. In this work, we disclose eigenvectors of the DFT matrix inspired by the ideas behind bilinear transform. The bilinear transform maps the analog space to the discrete sample space. As $j \omega$ in the analog $s$-domain is mapped to the unit circle one-to-one without aliasing in the discrete $z$-domain, it is appropriate to use it in the discretization of the eigenfunctions of the Fourier transform. We obtain Hermite-Gaussian-like eigenvectors of the DFT matrix. For this purpose we propose three different methods and analyze their stability conditions. These methods include better conditioned commuting matrices and higher order methods. We confirm the results with extensive simulations.
\end{abstract}

\section{Introduction}

Discretization of the fractional Fourier transform (FrFT) is vital in many application areas including signal and image processing, filtering, sampling, and time-frequency analysis [1-3]. As FrFT is related to the Wigner distribution [1], it is a powerful tool for time-frequency analysis, for example, chirp rate estimation [4].

There have been numerous discrete fractional Fourier transform (DFrFT) definitions [5-11]. Santhanam and McClellan [5] define a DFrFT simply as a linear combination of powers of the DFT matrix. However, this definition is not satisfactory, since it does do not mimic the properties of the continuous FrFT.

Candan et al. [6] use the $S$ matrix, which has been introduced earlier by Dickinson and Steiglitz [12] to find the eigenvectors of the DFT matrix in order to generate a DFrFT matrix. The $S$ matrix commutes with the DFT matrix, which ensures that both of these matrices share at least one eigenvector set in common. This approach is based on the second-order Hermite-Gaussian generating differential equation. Candan et al. [6] simply replace the derivative operator with the second-order discrete Taylor approximation to second derivative and the Fourier operator with the DFT matrix.

Pei et al. [7] define a commuting $\mathbf{T}$ matrix inspired by the work of Grünbaum [13], whose eigenvectors approximate the samples of continuous Hermite-Gaussian functions better than the eigenvectors of $\mathbf{S}$. Furthermore, the authors use linear combinations of $\mathbf{S}$ and $\mathbf{T}$ matrices as $\mathbf{S}+k \mathbf{T}$ to furnish the basis of eigenvectors for the DFrFT matrix.

Candan introduces $\boldsymbol{S}_{k}$ [8] matrices whose eigenvectors are higher-order approximations to the Hermite-Gaussian functions. The idea is to employ higher-order Taylor series approximations to the derivative operator, which replaces the second derivative operator in the Hermite-Gaussian generating differential equation. However, the order of approximation $k$ is limited by the dimension of the $\boldsymbol{S}_{k}$ matrix $2 k+1 \leq N$.

Pei et al. [10] recently removed the upper bound of this approximation and obtained higher-order approximations. However, it needs high computational cost to generate Pei's $S_{k}$ matrices. More recently, in [14] the authors present the closed form of $\boldsymbol{S}_{k}$ matrix as $k \rightarrow \infty$ in the limit.

In this work, we find eigenvectors of the DFT matrix in a completely different way. We define the derivative operator 
as its bilinear discrete equivalent to discretize the HermiteGaussian differential equation. Since the bilinear transform maps the analog domain to the discrete domain one-to-one, we find eigenvectors which are close to the samples of the Hermite-Gaussian functions. We also analyze the stability issues. Additionally, two more methods are proposed, which employ better conditioned and higher-order bilinear matrices.

The paper is organized as follows. Section 2 gives introductory information on Hermite-Gaussian functions, basics of how to generate commuting matrices and the bilinear transform. Section 3 presents the proposed methods by defining the bilinear transform-based commuting matrices including the stability analysis. Simulation results and performance analysis are given in Section 4. The paper concludes in Section 5.

\section{Preliminaries}

2.1. Hermite-Gaussian Functions. The Hermite-Gaussian functions span the space of Hilbert space $L^{2}(R)$ of square integrable functions, which are well localized in both time and frequency domains. These functions are defined by a Hermite polynomial modulated with a Gaussian function

$$
\Psi_{m}(t)=\frac{2^{1 / 4}}{\sqrt{2^{m} m !}} H_{m}(\sqrt{2 \pi} t) e^{-\pi t^{2}}
$$

where $H_{m}(t)$ is the $m$ th-order Hermite polynomial. HermiteGaussian functions are eigenfunctions of the Fourier transform

$$
\mathcal{F}\left\{\Psi_{m}\right\}(t)=e^{-\jmath m \pi / 2} \Psi_{m}(t),
$$

where $\mathcal{F}$ is the Fourier transformation operator and $e^{-\jmath m \pi / 2}$ is the $m$ th-order eigenvalue. An $m$ th-order HermiteGaussian function has $m$ zero-crossings. The HermiteGaussian functions are homogeneous solutions of the differential equation, which is also known as the HermiteGaussian generating differential equation

$$
\frac{\mathrm{d}^{2} f(t)}{\mathrm{d} t^{2}}-4 \pi^{2} t^{2} f(t)=\lambda f(t),
$$

with $\lambda=2 m+1$. The Hermite-Gaussian generating function can be expressed by its operator equivalent as

$$
\left\{\mathscr{D}^{2}+\mathcal{F} \mathscr{D}^{2} \mathcal{F}^{-1}\right\} f(t)=\lambda f(t),
$$

where $\mathscr{D}^{2}$ denotes the second derivative operator.

2.2. Commuting Matrix Generation. Let $\mathbf{A}$ and $\mathbf{B}$ be $N \times N$ square matrices. If $\mathbf{A B}=\mathbf{B A}$, then $\mathbf{A}$ and $\mathbf{B}$ are commuting matrices. If $\mathbf{A}$ and $\mathbf{B}$ commute, they share at least one set of common eigenvector sets [6].

Candan [8] showed that a DFT commuting matrix $\mathbf{K}$ can be obtained for any arbitrary $N \times N$ matrix $\mathbf{L}$ as

$$
\mathbf{K}=\mathbf{L}+\mathbf{F L F}^{-1}+\mathbf{F}^{2} \mathbf{L F}^{-2}+\mathbf{F}^{3} \mathbf{L F}^{-3},
$$

where $\mathbf{F}$ is the $N$ point DFT matrix which is defined as

$$
(\mathbf{F})_{n, m}=\frac{1}{\sqrt{N}} \exp \left(-\jmath \frac{2 \pi}{N} n m\right), \quad n, m=0,1, \ldots, N-1 .
$$

In [10] it is shown that if $\mathbf{L}$ commutes with $\mathbf{F}^{2},(5)$ is simplified to

$$
\mathbf{K}=\mathbf{L}+\mathbf{F L F}^{-1} .
$$

Theorem 1. One can further extend this idea such that, if $\mathbf{L}$ is circulant and symmetric the above equation is also valid.

Proof. Let $\mathbf{C}$ be a circulant and symmetric matrix, then the eigenvalue decomposition of $\mathbf{C}$ is [15, pages 201-202]

$$
\mathbf{C}=\mathbf{F}^{-1} \Lambda_{C} \mathbf{F},
$$

where $\boldsymbol{\Lambda}_{C}=\operatorname{diag}(\sqrt{N} \mathbf{F c})$ is a diagonal matrix containing eigenvalues of $\mathbf{C}$. Here, $\mathbf{c}$ is the first column of $\mathbf{C}$, and $N$ is the dimensional of $\mathbf{C}$. As $\mathbf{C}$ is symmetric, the above equation is equivalent to

$$
\mathbf{C}=\mathbf{F} \Lambda_{C} \mathbf{F}^{-1}
$$

since the symmetry implies that $\mathbf{C}^{T}=\mathbf{C}$. Hence we can conclude that $\mathbf{C}+\mathbf{F C F}^{-1}=\mathbf{F}^{2} \mathbf{C F}^{-2}+\mathbf{F}^{3} \mathbf{C F}^{-3}$ when we replace (9) in the left hand side and (8) in the right hand side of this equation. Consequently, the proof of

$$
\mathbf{K}=\mathbf{C}+\mathbf{F C F}^{-1}
$$

is complete. We can conclude that while generating DFT commuting matrices, a good choice is to chose real, symmetric and circulant matrices and replace them with $\mathbf{C}$ in (10).

2.3. Bilinear Transform. Bilinear transform is a useful and popular tool in signal and system analysis, which is often used to map the Laplace $s$-domain to the $z$-domain. There are numerous finite difference approximation (FDA) methods for this mapping. The most popular ones are the forward and backward difference methods and the bilinear transform. The forward difference method discretizes the derivative operator by mapping $\mathrm{d} x(t) / \mathrm{d} t \Rightarrow(x(n)-x(n-1)) / \Delta t$ whereas the backward difference method impose $\mathrm{d} x(t)) \mathrm{d} t \Rightarrow(x(n+$ 1) $-x(n)) / \Delta t$.

The bilinear transform defines the discrete differentiation of a signal $x(n)$ as

$$
x^{\prime}(n)+x^{\prime}(n-1)=\frac{c}{\Delta t}(x(n)-x(n-1)),
$$

where $x^{\prime}(n)$ is the discrete derivative of $x(n), \Delta t=1 / \sqrt{N}$ is the sampling period, $N$ is the length of the signal $x(n)$, and $c$ is a real scalar. Hence, the second-order discrete derivative $x^{\prime \prime}(n)$ can be defined through the centered form expression

$$
\begin{aligned}
x^{\prime \prime}(n-1)+2 x^{\prime \prime}(n)+x^{\prime \prime}(n+1) \\
\quad=\left(\frac{c}{\Delta t}\right)^{2}(x(n-1)-2 x(n)+x(n+1)) .
\end{aligned}
$$


The bilinear transform maps the analog domain to the discrete domain one-to-one. It maps points in the $s$-domain with $\operatorname{Re}\{s\}=0$ ( $j \omega$ axis) to the unit circle in the $z$-plane $|z|=1$. However, the forward difference method maps the $j \omega$ to a circle of radius 0.5 and centered at the point $z=0.5$ as shown in Figure 1. Bilinear transform maps every point in the $j \omega$-plane to the $z$-plane without aliasing.

We express (12) in matrix form as

$$
\mathbf{B}_{1} \mathbf{X}^{\prime \prime}=\left(\frac{c}{\Delta t}\right)^{2} \mathbf{E}_{2} \mathbf{X}
$$

where $\mathbf{X}^{\prime \prime}=\left[x^{\prime \prime}(0), x^{\prime \prime}(1), \ldots, x^{\prime \prime}(N-1)\right]^{T}, \mathbf{X}=$ $[x(0), x(1), \ldots, x(N-1)]^{T}$ with

$$
\begin{gathered}
\mathbf{B}_{1}=\left[\begin{array}{cccccc}
2 & 1 & 0 & \cdots & 0 & 1 \\
1 & 2 & 1 & \cdots & & 0 \\
0 & 1 & 2 & \ddots & & \vdots \\
\vdots & & \ddots & \ddots & \ddots & \vdots \\
\vdots & & & \ddots & \ddots & 1 \\
1 & 0 & 0 & \cdots & 1 & 2
\end{array}\right], \\
\mathbf{E}_{2}=\left[\begin{array}{cccccc}
-2 & 1 & 0 & \cdots & 0 & 1 \\
1 & -2 & 1 & \cdots & & 0 \\
0 & 1 & -2 & \ddots & & \vdots \\
\vdots & & \ddots & \ddots & \ddots & \vdots \\
\vdots & & & \ddots & \ddots & 1 \\
1 & 0 & 0 & \cdots & 1 & -2
\end{array}\right] .
\end{gathered}
$$

Hence, we conclude with an equivalent form of discrete second derivative as

$$
\mathbf{X}^{\prime \prime}=\left(\frac{c}{\Delta t}\right)^{2} \mathbf{B}_{1}^{-1} \mathbf{E}_{2} \mathbf{X}
$$

with the discrete second derivative operator $\mathbf{D}^{2}=$ $(c / \Delta t)^{2} \mathbf{B}_{1}^{-1} \mathbf{E}_{2}$.

\section{Obtaining DFT Commuting Matrices}

An easy and accurate way of obtaining Hermite-Gaussianlike eigenvectors of the DFT matrix is to define a better commuting matrix, which imitates the Hermite-Gaussian generating differential equation given in (3) as a discrete substitute. In this section we disclose an elegant way of obtaining better commuting matrices by taking advantage of the bilinear transform, which is a good discrete substitute for the derivative operator.

The algorithm is straightforward; we substitute the second derivative and the Fourier transform operators in (3) with the matrix given in (16) and the DFT matrix,

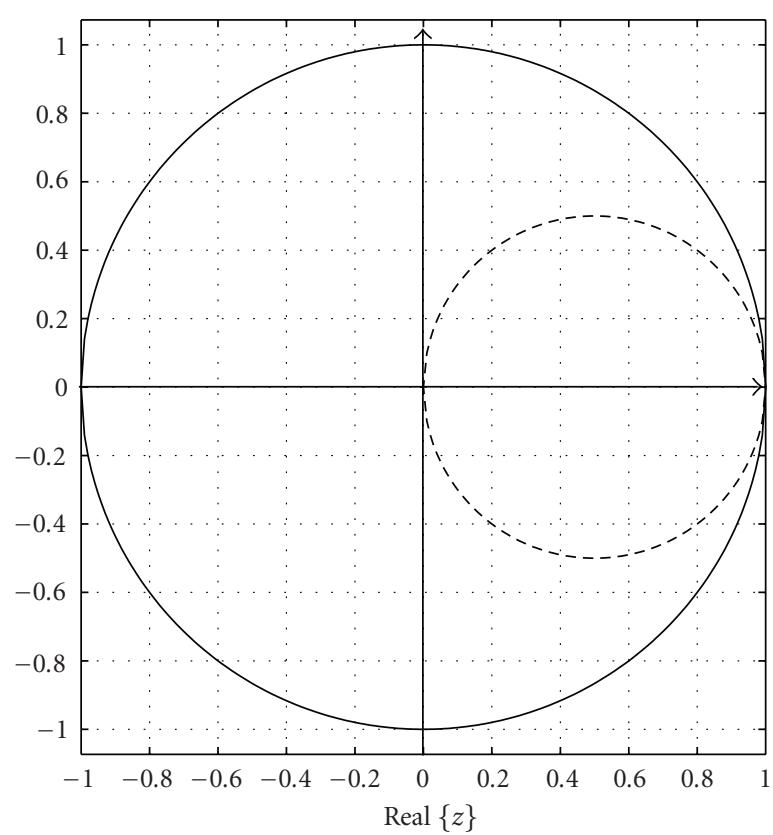

FIGURE 1: Image of $j \omega$ axis in the $z$-plane for bilinear transform and the forward difference method. Solid: bilinear transform, dashed: forward difference method.

respectively. Hence, DFT commuting matrix inspired by the bilinear transform is given by

$$
\mathbf{B}=\mathbf{B}_{1}^{-1} \mathbf{E}_{2}+\mathbf{F B}_{1}^{-1} \mathbf{E}_{2} \mathbf{F}^{-1} .
$$

We omit the coefficient $(c / \Delta t)^{2}$, since it has no effect on the eigenvectors of $\mathbf{B}$.

\section{Theorem 2. B commutes with the DFT matrix.}

Proof. As $\mathbf{B}_{1}$ and $\mathbf{E}_{2}$ are both circulant and symmetric, $\mathbf{B}_{1}^{-1} \mathbf{E}_{2}$ is symmetric and circulant also. We use Theorem 1 given in Section 2.2, which states that any circulant and symmetric matrix $\mathbf{C}$ can be used to generate a commuting matrix as in (10). Since $\mathbf{B}_{1}^{-1} \mathbf{E}_{2}$ is both circulant and symmetric, the proof is complete.

After generating the commuting matrix $\mathbf{B}$, we find its eigenvectors. The eigenvectors are Hermite-Gaussian-like eigenvectors with the number of zero-crossings equal to the order of Hermite-Gaussian eigenvectors. In Section 4 we give extensive simulations and results on these Hermite-Gaussian like eigenvectors.

3.1. Stability. Stability of $\mathbf{B}$ can easily be proved when $\mathbf{B}_{1}$ is not singular. We can show this by the eigenvalue decomposition of $\mathbf{B}_{1}$

$$
\mathbf{B}_{1}=\mathbf{F}^{-1} \boldsymbol{\Lambda}_{B_{1}} \mathbf{F},
$$

where $\Lambda_{B_{1}}$ is a diagonal matrix containing the eigenvalues of $\mathbf{B}_{1}$. As $\mathbf{B}_{1}$ is circulant, the eigenvalues are $\boldsymbol{\Lambda}_{B_{1}}=$ $\operatorname{diag}\left(\sqrt{N} \mathbf{F} \mathbf{b}_{1}\right)$, where $\mathbf{b}_{1}$ is the first column of $\mathbf{B}_{1}$ and $N \times N$ 
is the dimension of $\boldsymbol{\Lambda}_{B_{1}}$. As $\mathbf{b}_{1}=[2,1,0,0, \ldots, 1]^{T}$, Fourier transform of $\mathbf{b}_{1}$ can be easily found and replaced to find the eigenvalues $\boldsymbol{\Lambda}_{B_{1}}$

$$
\boldsymbol{\Lambda}_{B_{1}}=\operatorname{diag}\left(2+2 \cos \left(\frac{2 \pi n}{N}\right)\right), \quad n=0,1,2, \ldots, N-1 .
$$

$\boldsymbol{\Lambda}_{B_{1}}$ is never zero for odd $N$, since $\operatorname{diag}(2+2 \cos (2 \pi n / N))>$ 0 for all $n$. However as $N$ increases $\Lambda_{B_{1}}$ becomes poorly conditioned. Besides, for even $N$, $\operatorname{diag}(2+2 \cos (2 \pi n / N))=0$ for $n=N / 2$, which causes instability. We can add a small $\xi>0$ in the diagonal of $\mathbf{B}_{1}$ to overcome instability. Then $\boldsymbol{\Lambda}_{B_{1}}$ is changed to

$$
\boldsymbol{\Lambda}_{B_{1}}=\operatorname{diag}\left(2+\xi+2 \cos \left(\frac{2 \pi n}{N}\right)\right), \quad n=0,1,2, \ldots, N-1
$$

to preserve stability for even $N$. Consequently, to ensure stability we substitute $\mathbf{B}_{1}$ defined in (14) with

$$
\widehat{\mathbf{B}}_{1}=\left[\begin{array}{cccccc}
2+\xi & 1 & 0 & \cdots & 0 & 1 \\
1 & 2+\xi & 1 & \cdots & & 0 \\
0 & 1 & 2+\xi & \ddots & & \vdots \\
\vdots & & \ddots & \ddots & \ddots & \vdots \\
\vdots & & & \ddots & \ddots & 1 \\
1 & 0 & 0 & \cdots & 1 & 2+\xi
\end{array}\right]
$$

Adding a small $\xi$ value in the diagonal will not perturb the eigenvectors of the commuting matrix.

3.2. Better Conditioned Bilinear Methods. Bilinear transform can be considered as a trapezoidal approach to the derivative. Hence, we can assure stability by using alternative $\mathbf{B}_{1}$ matrices. We have found out that changing the diagonal of $\mathbf{B}_{1}$ by a constant $k>2$ both ensures the stability and increases the performance. Therefore we substitute $\mathbf{B}_{1}$ with $\overline{\mathbf{B}}_{1}$, where we define $\overline{\mathbf{B}}_{1}$ as

$$
\overline{\mathbf{B}}_{1}=\left[\begin{array}{cccccc}
k & 1 & 0 & \cdots & 0 & 1 \\
1 & k & 1 & \cdots & & 0 \\
0 & 1 & k & \ddots & & \vdots \\
\vdots & & \ddots & \ddots & \ddots & \vdots \\
\vdots & & & \ddots & \ddots & 1 \\
1 & 0 & 0 & \cdots & 1 & k
\end{array}\right] .
$$

As $k>2$, the commuting matrix is better conditioned. The optimum value of $k$ is found to be approximately 4.3 , which is given in Section 4.

\subsection{Higher-order Bilinear Differentiation Matrix Substitutes.} So far, we have used the bilinear-transform-inspired matrices to find a better discrete substitute for the second derivative. To find better definitions of differentiation matrices we suggest that a Taylor series-like approach to $\overline{\mathbf{B}}_{1}$
TABLE 1: Optimum $a_{i}$ coefficients generated for $\overline{\mathbf{B}}_{14}$.

\begin{tabular}{lc}
\hline$a_{i}$ & Optimum Value \\
\hline$a_{1}$ & 1.00 \\
$a_{2}$ & 0.247634068038315 \\
$a_{3}$ & -0.103839534211561 \\
$a_{4}$ & -0.141176982675410 \\
$a_{5}$ & 0.005956945393076 \\
$a_{6}$ & -0.008133047918379 \\
$a_{7}$ & -0.020103743248487 \\
$a_{8}$ & -0.001866823892062 \\
$a_{9}$ & -0.000336065416294 \\
$a_{10}$ & -0.002383849560258 \\
$a_{11}$ & -0.000725049220057 \\
$a_{12}$ & -0.000698349278537 \\
$a_{13}$ & -0.003339855815284 \\
$a_{14}$ & -0.001759635742928 \\
\hline
\end{tabular}

(1) Compute one of $\widehat{\mathbf{B}}_{1}, \overline{\mathbf{B}}_{1}$, or $\overline{\mathbf{B}}_{n}$ matrices.

(2) Replace the computed matrix in (17) as a substitute for $\mathbf{B}_{1}$ and compute the DFT-commuting matrix $\mathbf{B}$.

(3) Find the eigenvectors of $\mathbf{B}$, which are HermiteGaussian-like orthonormal vectors.

Algorithm 1: Summary of the proposed algorithms.

will grant us higher-order bilinear differentiation matrices. Therefore, we define higher-order bilinear differentiation matrices as

$$
\overline{\mathbf{B}}_{n}=a_{1} \overline{\mathbf{B}}_{1}+a_{2}\left(\overline{\mathbf{B}}_{1}\right)^{2}+\cdots+a_{n}\left(\overline{\mathbf{B}}_{1}\right)^{n},
$$

where we name $\overline{\mathbf{B}}_{n}$ as $n$ th-order bilinear approximation to the second derivative, and $a_{i}$ are real scalars. The value of $k=$ 4.3 is chosen for $\overline{\mathbf{B}}_{n}$, as it is an optimum value with respect to minimum total error norm which is discussed in Section 4. We have not come up with an analytical expression of $a_{i}$ 's yet, however, genetic and/or pattern search algorithms may be used to optimize the coefficients.

We have used the genetic [16] and the pattern search [17] algorithms and determined optimum $a_{i}$ coefficients, $i=1,2, \ldots, 14$, which are given in Table 1 . These coefficients are inserted in (23) to obtain $\overline{\mathbf{B}}_{14}$. We have generated the commuting matrix $\mathbf{B}$ by substituting $\mathbf{B}_{1}$ with $\overline{\mathbf{B}}_{14}$ in (17). When $\overline{\mathbf{B}}_{14}$ is employed, eigenvectors of $\mathbf{B}$ are found to be very close to the samples of Hermite-Gaussian functions as the performance is discussed in detail in the very Section 4 .

So far, three different methods are proposed, which are summarized in Algorithm 1. The first method computes $\widehat{\mathbf{B}}_{1}$, in which a small $\xi$ is added in the diagonal of $\mathbf{B}_{1}$ to achieve stability. In the second method we alter the diagonal of $\overline{\mathbf{B}}_{1}$, with a value $k>2$. Changing the diagonal both improves the performance and ensures stability. In the last proposed method we find higher-order matrices, using the $\overline{\mathbf{B}}_{1}$ and its weighted powers with $k=4.3$ for a better definition of the commuting matrix. Afterwards, we replace the computed $\hat{\mathbf{B}}_{1}, \overline{\mathbf{B}}_{1}$, or $\overline{\mathbf{B}}_{n}$ with $\mathbf{B}_{1}$ in (17). The obtained matrix $\mathbf{B}$ is the DFT-commuting 


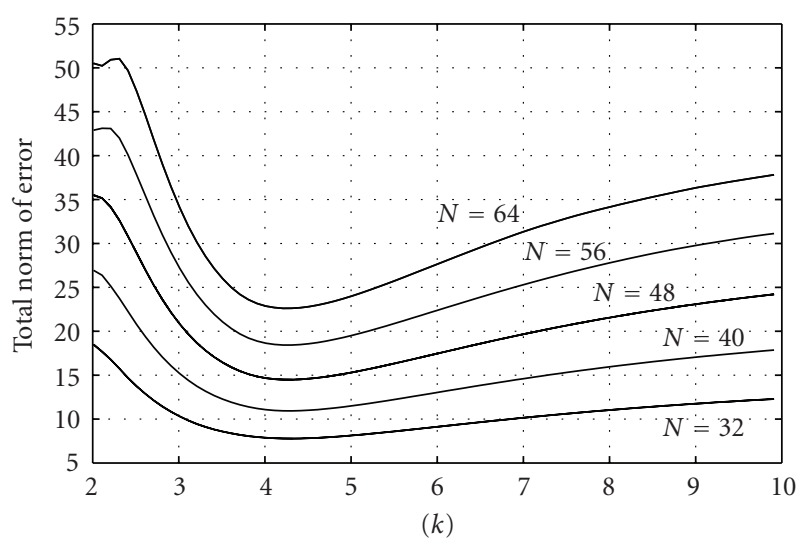

(a)

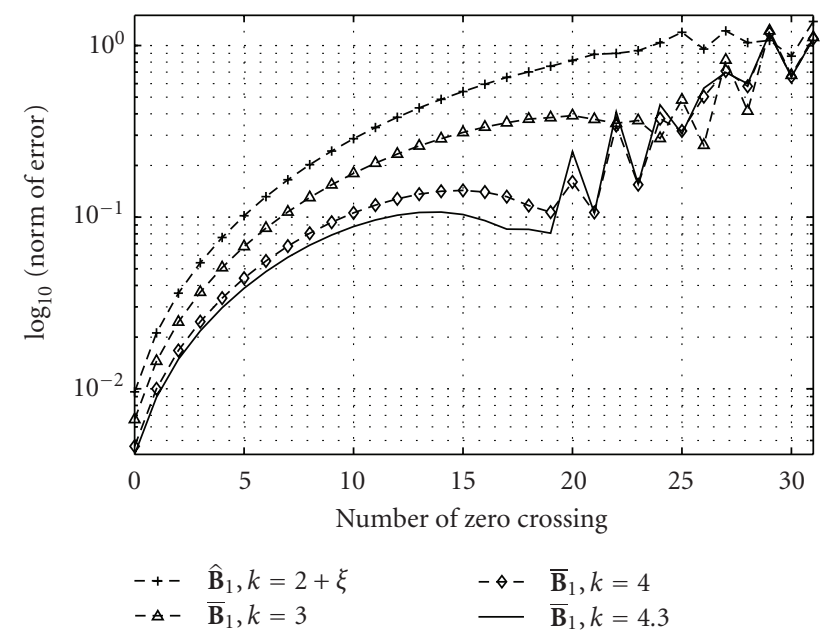

(b)

Figure 2: (a) The total norm of error versus $k$ in $\overline{\mathbf{B}}_{1}$ for $N=32$, $40,48,56$, and $k=4.3$. The total norm of error is minimum when $k \approx 4.3$. (b) Error norms between the discrete Hermite-Gaussian like eigenvectors and the samples of the Hermite-Gaussian functions when $\overline{\mathbf{B}}_{1}$ for $k=3,4$, and $k=4.3$ and $\hat{\mathbf{B}}_{1}$ with $N=32$.

matrix whose eigenvectors are the Hermite-Gaussian-like orthonormal vectors.

\section{Simulations and Results}

We have proposed three different techniques for finding Hermite-Gaussian-like eigenvectors of the DFT. In the first method we employ $\widehat{\mathbf{B}}_{1}$ defined in (21). As a second method we use $\overline{\mathbf{B}}_{1}$ as defined in (22) for different values of $k$. Finally, we employ $\overline{\mathbf{B}}_{n}$ given in (23). We replace each matrices in (17) as a substitute to $\mathbf{B}_{1}$ to generate commuting matrices $\mathbf{B}$. Afterwards, we find eigenvectors of these commuting matrices and find the norm of error between samples of corresponding Hermite-Gaussian functions and the eigenvectors.

First we compare total norms of errors between the Hermite-Gaussian functions and the samples of HermiteGaussian-like eigenvectors to determine optimum $k$ for $\overline{\mathbf{B}}_{1}$. We define the total norm of error as sum of norms of error for each eigenvector. Figure 2(a) shows the total norm of error

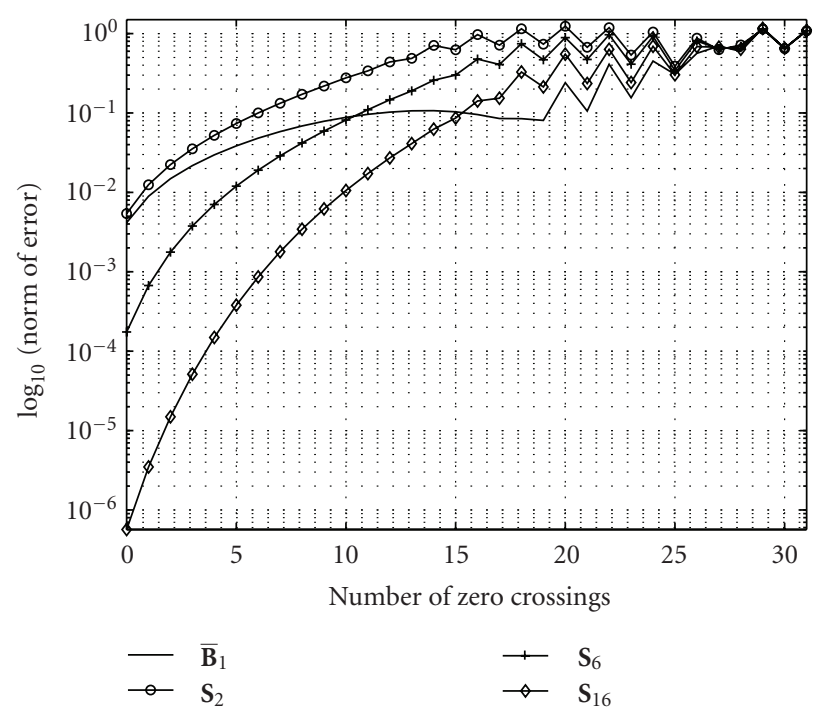

(a)

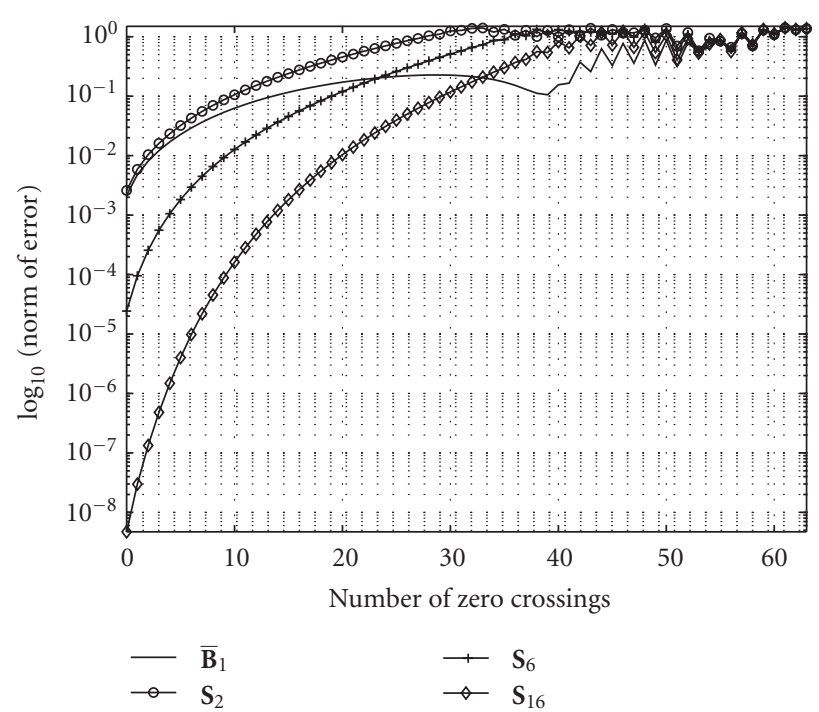

(b)

FIGURE 3: Error norms between the discrete Hermite-Gaussian like eigenvectors and the samples of the Hermite-Gaussian functions of $\overline{\mathbf{B}}_{1}$ method when $k=4.3$ are compared with various other methods for (a) $N=32$ and (b) $N=64$.

versus $k$ for different values of $N$, and the best value for $k$ is approximately 4.3 .

Comparison of errors between $\overline{\mathbf{B}}_{1}$ for $k=3$, 4 , and 4.3 and $\widehat{\mathbf{B}}_{1}$ with the dimensional $N=32$ is given in Figure 2(b). The error norm is measured as the norm of error between the samples of Hermite-Gaussian functions and HermiteGaussian like eigenvectors of $\mathbf{B}$ using these matrices. As it is clear from the figures, the best overall performance is obtained in the $\overline{\mathbf{B}}_{1}$ method when $k=4.3$.

Figure 3(a) plots the norms of errors for different methods defined in [8]. We compare the error norms of $\mathbf{S}_{2}, \mathbf{S}_{6}$, and $\mathbf{S}_{16}$ in between, which are of $O\left(h^{2}\right), O\left(h^{6}\right)$, and $O\left(h^{16}\right)$ Taylor approximations, respectively, as shown in [8], with the $\overline{\mathbf{B}}_{1}$ method, $k=4.3$ for $N=32$. Figure $3(\mathrm{~b})$ plots 


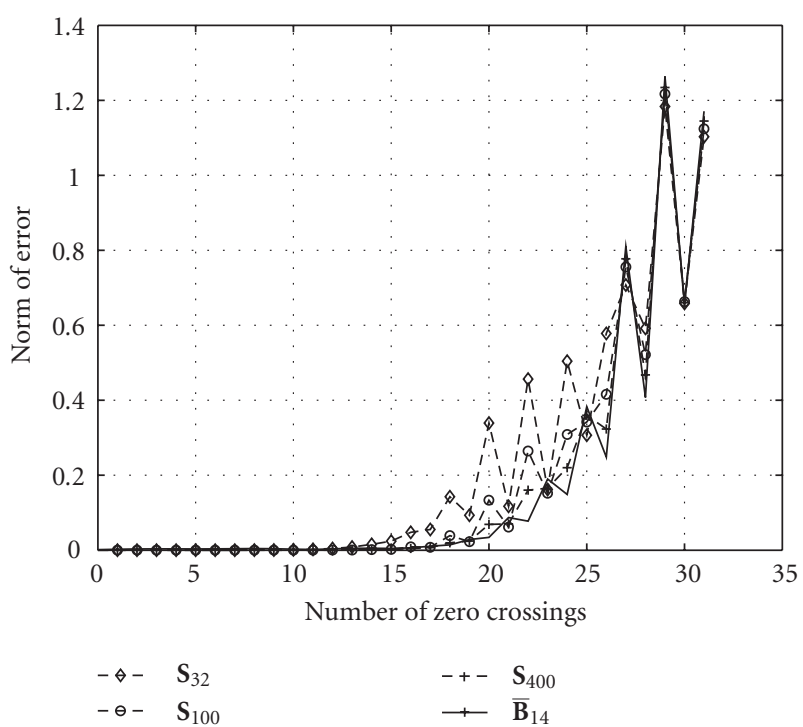

Figure 4: Comparison of error norms between the discrete Hermite-Gaussian like eigenvectors and the samples of the Hermite-Gaussian functions of $\overline{\mathbf{B}}_{14}$ and $\boldsymbol{S}_{32}, \boldsymbol{S}_{100}$, and $\boldsymbol{S}_{400}$ methods for $N=32$.

the same comparison for $N=64$. These plots show that our proposed algorithm is slightly worse than some other methods for small orders, but much better for higher-orders of eigenvectors. As it is clear from the figures, our method outperforms the other methods in total.

We compare the proposed higher-order $\overline{\mathbf{B}}_{14}$ method with the other higher-order methods, $\mathbf{S}_{32}, \boldsymbol{S}_{100}$, and $\boldsymbol{S}_{400}$ that employ higher-order Taylor approximations to the second derivative as shown in [10]. Figure 4 presents the performance of the proposed method together with the other methods. Despite the fact that our method uses only the 14th order approximation, it is definitely better than these methods, even better than $\boldsymbol{S}_{400}$.

\section{Conclusions}

As the eigenvectors that are closer to the samples of continuous Hermite-Gaussian functions are important for a better definition of DFrFT, we employ bilinear transformbased methods to define better commuting matrices. We have proposed three different methods and analyzed their stability issues. A stable method is proposed by inserting a small $\xi$ in the diagonal of the bilinear matrix. Betterconditioned bilinear differentiation matrices that have better performance are also obtained. Besides, a method of generating higher-order bilinear differentiating matrices is also suggested.

Simulation results show that the proposed methods posess better eigenvectors when compared to the other methods recently suggested.

Future works on this subject may include finding a closed form expression for the coefficients generating the higher-order bilinear matrices, $\overline{\mathbf{B}}_{n}$. Furthermore, $\mathbf{B}_{n}$ matrices may be used in linear combinations with other commuting matrices, such as $\boldsymbol{S}_{2 k}$.

\section{References}

[1] H. M. Ozaktas, Z. Zalevski, and M. A. Kutay, The Fractional Fourier Transform with Applications in Optics and Signal Processing, John Wiley \& Sons, New York, NY, USA, 2001.

[2] M. A. Kutay, H. M. Ozaktas, O. Ankan, and L. Onural, "Optimal filtering in fractional Fourier domains," IEEE Transactions on Signal Processing, vol. 45, no. 5, pp. 1129-1143, 1997.

[3] X.-G. Xia, "On bandlimited signals with fractional Fourier transform," IEEE Signal Processing Letters, vol. 3, no. 3, pp. 7274, 1996.

[4] O. Akay and G. F. Boudreaux-Bartels, "Fractional convolution and correlation via operator methods and an application to detection of linear FM signals," IEEE Transactions on Signal Processing, vol. 49, no. 5, pp. 979-993, 2001.

[5] B. Santhanam and J. H. McClellan, "The discrete rotational Fourier transform," IEEE Transactions on Signal Processing, vol. 44, no. 4, pp. 994-998, 1996.

[6] Ç. Candan, M. A. Kutay, and H. M. Ozaktas, "The discrete fractional Fourier transform," IEEE Transactions on Signal Processing, vol. 48, no. 5, pp. 1329-1337, 2000.

[7] S.-C. Pei, W.-L. Hsue, and J.-J. Ding, "Discrete fractional Fourier transform based on new nearly tridiagonal commuting matrices," IEEE Transactions on Signal Processing, vol. 54, no. 10, pp. 3815-3828, 2006.

[8] Ç. Candan, "On higher order approximations for HermiteGaussian functions and discrete fractional Fourier transforms," IEEE Signal Processing Letters, vol. 14, no. 10, pp. 699702, 2007.

[9] S.-C. Pei, J.-J. Ding, W.-L. Hsue, and K.-W. Chang, "Generalized commuting matrices and their eigenvectors for DFTs, offset DFTs, and other periodic operations," IEEE Transactions on Signal Processing, vol. 56, no. 8, pp. 3891-3904, 2008.

[10] S.-C. Pei, W.-L. Hsue, and J.-J. Ding, "DFT-commuting matrix with arbitrary or infinite order second derivative approximation," IEEE Transactions on Signal Processing, vol. 57, no. 1, pp. 390-394, 2009.

[11] B. Santhanam and T. S. Santhanam, "Discrete Gauss-Hermite functions and eigenvectors of the centered discrete Fourier transform," in Proceedings of the IEEE International Conference on Acoustics, Speech and Signal Processing ( ICASSP '07), vol. 3, pp. 1385-1388, 2007.

[12] B. W. Dickinson and K. Steiglitz, "Eigenvectors and functions of the discrete Fourier transform," IEEE Transactions on Acoustics, Speech, and Signal Processing, vol. 30, no. 1, pp. 2531, 1982.

[13] F. A. Grünbaum, "The eigenvectors of the discrete Fourier transform: a version of the Hermite functions," Journal of Mathematical Analysis and Applications, vol. 88, no. 2, pp. 355363, 1982.

[14] A. Serbes and L. Durak, "Efficient computation of DFT commuting matrices by a closed-form infinite order approximation to the second differentiation matrix," Signal Process. In press.

[15] G. G. Golub and C. F. Van Load, Matrix Computations, The Johns Hopkins University Press, London, UK, 3rd edition, 1993. 
[16] D. E. Goldberg, Genetic Algorithms in Search, Optimzation and Machine Learning, Addison-Wesley, Reading, Mass, USA, 1989.

[17] C. Audet and J. E. Dennis Jr., "Analysis of generalized pattern searches," SIAM Journal on Optimization, vol. 13, no. 3, pp. 889-903, 2003. 